\title{
Activated pulmonary tuberculosis in a patient with melanoma during PD-I inhibition: a case report
}

\author{
Wan $\mathrm{He}^{1, *}$ \\ Xiangmei Zhang ${ }^{2, *}$ \\ Wenwen $\mathrm{Li}^{\prime}$ \\ Cheng Kong' \\ Yuanyang Wang ${ }^{3}$ \\ Lianyu Zhu' \\ Ruilian $X u^{\prime}$ \\ Guofang Deng ${ }^{4}$ \\ Peize Zhang ${ }^{4}$ \\ 'Department of Oncology, The Second \\ Clinical Medical College, Shenzhen \\ People's Hospital, Jinan University, \\ Shenzhen, Guangdong 518020, China; \\ ${ }^{2}$ Department of Pathology, The \\ Third People's Hospital of Shenzhen, \\ Shenzhen, Guangdong 5181 I2, China; \\ ${ }^{3}$ Department of Surgery, The Second \\ Clinical Medical College, Shenzhen \\ People's Hospital, Jinan University, \\ Shenzhen, Guangdong 518020, \\ China; ${ }^{4}$ Department of Pulmonary \\ Medicine, The Third People's Hospital \\ of Shenzhen, Shenzhen, Guangdong \\ $5|8| \mid 2$, China \\ *These authors contributed equally \\ to this work
}

This article was published in the following Dove Press journal:
OncoTargets and Therapy

\begin{abstract}
Background: PD-1 checkpoint inhibitors have shown a robust tumor response in the treatment of various cancers. Pembrolizumab is an anti-PD-1 checkpoint antibody approved for the treatment of unresectable or metastatic melanoma in more than 40 countries. Although autoimmune pneumonitis is considered a common immune-related adverse event of PD-1 inhibitors, only limited studies have assessed the development of opportunistic infections such as pulmonary tuberculosis (TB). Case presentation: A patient with metastatic melanoma whose pulmonary TB was activated after administration of pembrolizumab for melanoma is reported. Anti-TB drugs were administered, followed by pembrolizumab ( $2 \mathrm{mg} / \mathrm{kg}$, repeated every 28 days), which successfully cured the TB and achieved complete response for melanoma.

Conclusion: Activated pulmonary TB was observed during the administration of pembrolizumab. It was safe and effective in the current patient to combine anti-TB drugs and PD-1 inhibitors. More importantly, screening pulmonary TB before administration of PD-1 inhibitors is recommended. Keywords: checkpoint inhibitor, PD-1, pembrolizumab, metastatic melanoma, pulmonary tuberculosis, anti-tuberculosis treatment
\end{abstract}

\section{Background}

Pembrolizumab, one of the checkpoint inhibitors approved for unresectable or metastatic melanoma, has shown promising effects and results in prolonged overall survival. ${ }^{1}$ The most common immune-related adverse events include fatigue, rash, pruritus, arthralgia, amylase elevation, diarrhea, vitiligo, pneumonitis, hepatitis, and endocrine-related adverse events such as hyper- or hypothyroidism. ${ }^{2}$ However, patients with active infections, eg, tuberculosis (TB), were excluded from clinical trials of pembrolizumab for advanced melanoma. Thus, whether pembrolizumab improves or exacerbates TB is yet to be elucidated.

Currently, only one Caucasian case with melanoma and four cases of lung cancers or Hodgkin's lymphoma developing TB during treatment with PD-1 inhibitors have been reported. ${ }^{3-6}$ Herein, we report a patient with advanced melanoma developing active pulmonary TB during PD-1 inhibitor therapy. Different from other cases, this patient exhibited a complete response of metastatic tumor and negative conversion of liquid culture for bronchoalveolar lavage fluid (BALF) by combining anti-PD-1 and anti-TB treatment.

\section{Case presentation}

A 65 -year-old Chinese female patient presented a gradually increased mass $(4 \times 3 \mathrm{~cm})$ in the left thigh and three separated black skin lesions $(1 \times 1 \mathrm{~cm}$ or less $)$ on the left foot 
in December 2015. The resection of the mass in the left thigh and the largest skin lesion on the left foot was performed on December 4, 2015. The pathological exploration showed malignant melanoma with necrosis but no nerve or vascular invasion. The chest computed tomography (CT) scan (Figure 1A) showed a shadow at the posterior segment of the right upper lobe of the lung. However, neither respiratory nor systemic symptoms, such as cough, weight loss, fever, and night sweats, were observed. For the first-line treatment, high-dose IL-2 was the preferred treatment, as is the case for medically fit metastatic melanoma patients for $>20$ years. $^{7}$ Thus, IL-2 was administered to the patient at a dose of 64,000 $\mathrm{IU} / \mathrm{kg}$, qd, on days 1-10, repeated every 14 days for 6 cycles. However, a node in the left thigh around the surgical margin $(1.5 \times 1.5 \mathrm{~cm})$ and two new lymph nodes in the left groin $(2 \times 1$ $\mathrm{cm})$ were observed, indicating progressive disease. Another chest CT scan (Figure 1B), performed in March 2016, suggested a slightly reduced lesion of the right lobe of the lung.

Then, the patient initiated pembrolizumab treatment at a dose of $2 \mathrm{mg} / \mathrm{kg}$ every 3 weeks since June 2016. After 3 cycles of immunotherapy, all the nodes contracted remarkably. Nevertheless, the patient developed fatigue and dry mouth and skin, raising suspicion of Sjögren's syndrome with a positive concentration of the autoimmune antibody RO52 in the peripheral blood test. Thus, the administration of pembrolizumab at $2 \mathrm{mg} / \mathrm{kg}$ was continued at every 4 weeks for another 7 cycles until January 2017. The patient responded to pembrolizumab, and the nodes in the left thigh or groin, except for two black skin lesions at the bottom of left foot (decreasing in size from $0.5 \times 0.3$ to $0.2 \times 0.1 \mathrm{~cm}$ ), disappeared, as evaluated by ultrasonic scan in October 2016; however, tolerant oral ulcer, fatigue, and vitiligo were observed.

In February 2017, the patient presented about $10 \mathrm{~mL}$ of bloody sputum daily for a week without cough, night sweats, fever, or weight loss. The chest CT (Figure 1C) showed the increased size of the lesion in the right upper lobe without lymphadenopathy or pleural effusion. BALF was collected, and transbronchial lung biopsy was performed. Unconcentrated BALF was tested positive for GeneXpert Mycobacterium tuberculosis (MTB)/RIF with low semiquantitative value. Additionally, mutations in $r p o B$ gene that confer rifampicin resistance were not detected. The liquid culture and drug sensitivity test for BALF indicated MTB to be drug sensitive. Both examinations confirmed the diagnosis of active pulmonary TB. The transbronchial lung biopsy specimen showed a large amount of caseous necrosis (Figure 2A and B) with mediate lymphocyte infiltrate but few acid-fast bacilli. The number of CD4 and CD8 in the peripheral blood was significantly higher than the normal range $(1,544 / \mu \mathrm{L}$ and $712 / \mu \mathrm{L}$, respectively). Consequently, immunotherapy was paused and anti-TB drugs of isoniazid 0.3 , qd, rifampin 0.45 , qd, pyrazinamide 1.0 , qd, and
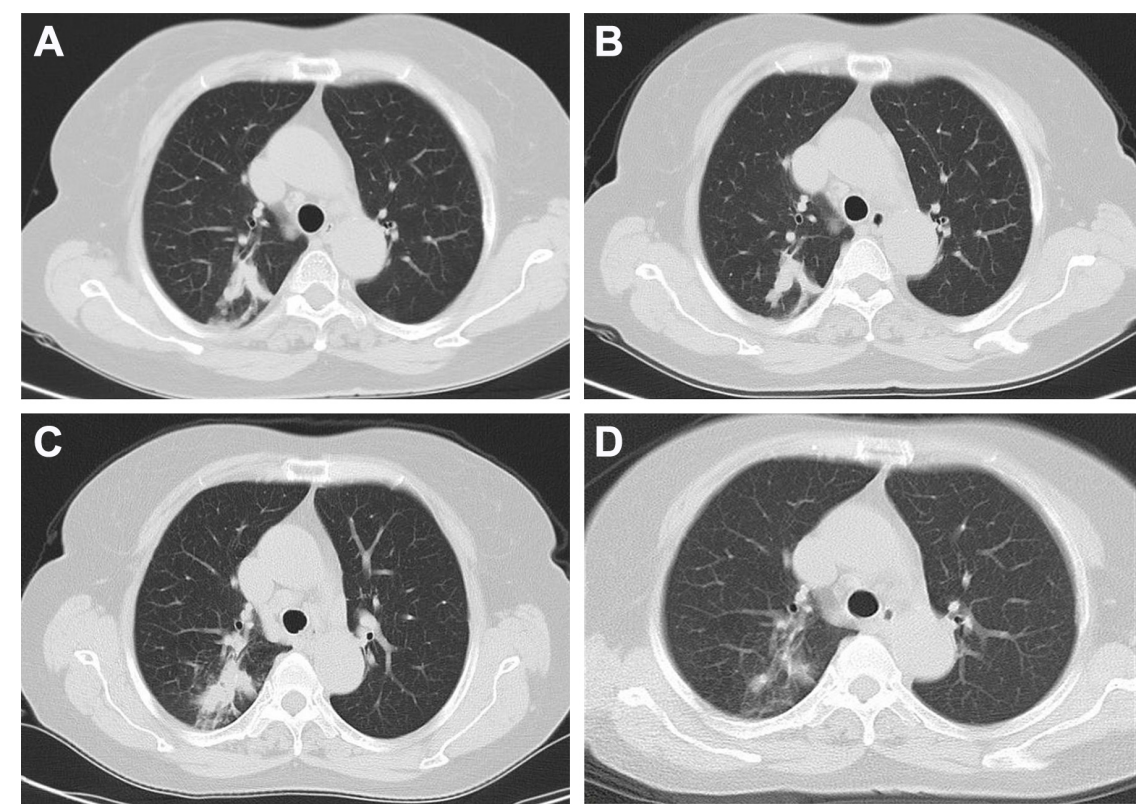

Figure I CT image.

Notes: (A) Infiltration of the right upper lobe of the lung before treatment. (B) Infiltration of the right upper lobe of the lung after IL-2 therapy. (C) Infiltration of the right upper lobe of lung worsened after PD-I inhibition. (D) Infiltration of the right upper lobe of lung absorbed after 6 weeks of anti-TB treatment.

Abbreviations: $\mathrm{CT}$, computed tomography; TB, tuberculosis. 

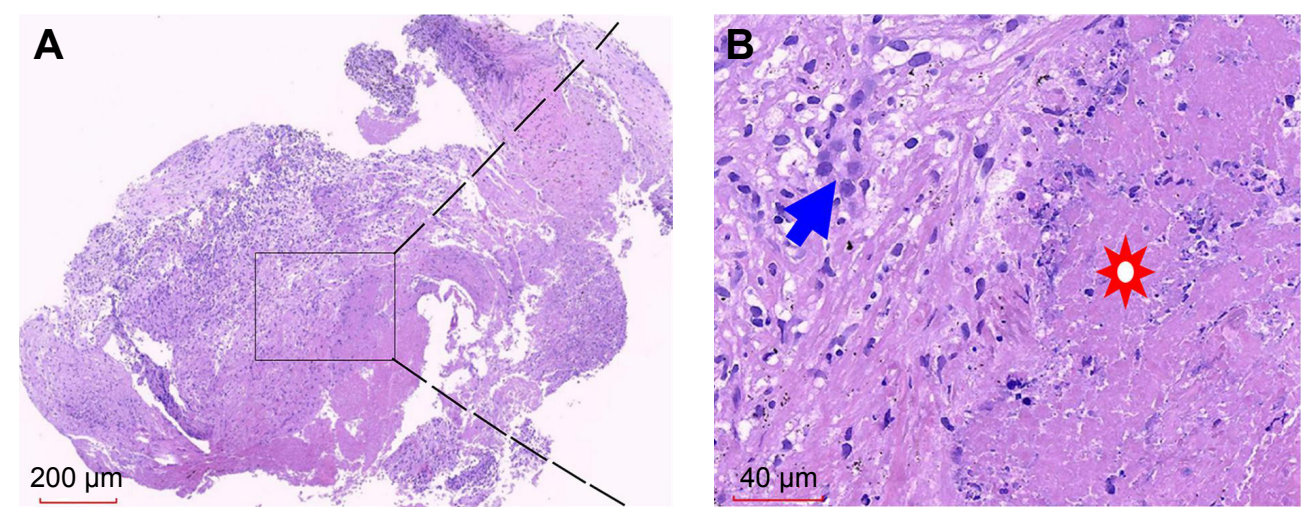

Figure 2 Histopathological findings of TB granuloma from the lung biopsy.

Notes: A large amount of caseous necrosis surrounded with epithelioid cells and diffused infiltrating lymphocytes (paraffin-embedded tissue by H\&E staining). (A) Original magnification (20x). (B) Local magnification of (A) (400x). Solar marking: caseous necrosis; blue arrows: epithelioid cells.

Abbreviation: TB, tuberculosis.

ethambutol 0.75, qd (HRZE) were administered according to the body weight. Bloody sputum ceased with anti-TB treatment 1-week posttreatment and the sputum culture for MTB was negative after 4 weeks. However, the patient complained of nausea and vomiting during the anti-TB treatment since week 4 , and the liver function test showed the abnormal level of alanine aminotransferase $240 \mathrm{U} / \mathrm{L}$, aspartate aminotransferase $277 \mathrm{U} / \mathrm{L}$, and total bilirubin $42 \mu \mathrm{mol} / \mathrm{L}$. Thus, the anti-TB treatment was suspended and liver-protecting drugs were administered. After 2 weeks, the liver function recovered and clinical symptoms improved significantly. HRZ was challenged again; however, the level of transaminases increased and isoniazid-induced fever was observed. Considering the negative sputum culture and side effects of HRZ, the administration of isoniazid, rifampin, and pyrazinamide was discontinued. The second-line anti-TB regimen, including streptomycin 0.75 , qd, ethambutol 0.75 , qd, and moxifloxacin 0.4 , qd, was administered for 4 months. The chest CT scan (Figure 1D) showed that the right pulmonary lesion had shrunk significantly in April 2017. Therefore, the patient was challenged with pembrolizumab monthly for 2 more cycles with the concurrent use of three anti-TB drugs. Combination of PD-1 inhibition and anti-TB treatment kept the patient fit with normal liver function and slightly improved dry mouth. Notably, no steroids were administered throughout the treatment. Then, the patient underwent radical resection of the remaining two black skin lesions at the bottom of the left foot on July 12, 2017, and histology did not show any tumor cells indicating a complete response. Subsequently, the patient was subjected to two consolidation treatments with pembrolizumab per month (total 14 cycles). Anti-TB therapy was discontinued in August 2017. Also, she was under medical surveillance every 3-6 months, and no recurrence was detected. Finally, the patient was under follow-up until the drafting of this case report.

\section{Consent for publication}

The study was approved by the Clinical Research Ethics Committee of Shenzhen People's Hospital, China. Written consent was obtained from the patient and her relatives for publication of the case report.

\section{Discussion}

Checkpoint inhibitors trigger an antitumor immune response by promoting T-cell activation to eliminate the tumor. The immune system activated by checkpoint inhibitors is effective in targeting pathogens such as hepatitis B virus, HIV, and bacteria. ${ }^{8}$ Active pulmonary TB is often developed in suppressed immune system induced by steroid administration or cytotoxic chemotherapy. ${ }^{9}$ To date, six cancer patients have been reported with activated TB during treatment with PD-1 inhibitors, including the current melanoma case. ${ }^{3-6}$ However, the mechanism underlying the development of TB during PD-1 inhibitor therapy is yet to be elucidated.

In mouse models, the survival of PD-1-deficient mice is decreased more significantly than wide-type ones after infection with TB. The loss or repression of PD-1 on CD4 T-cells sufficiently leads to rapid wasting and mortality during the clonal expansion phase of the T-cell response to MTB. ${ }^{10}$ Moreover, overinflammation and excessive necrosis were observed in biopsied lung tissue from PD-1 deficient mice. A high level of proinflammatory meditators and early recruitment of macrophages and neutrophils were found in the lung of PD-1 deficient mice. ${ }^{11}$ An increased number of $\mathrm{T}$ regulatory cells and decreased number of TB antigenspecific T-cells, which lead to persistent TB infection, were 
observed in PD-1 knockout mice. ${ }^{12}$ Among these studies, the overinflammation with a high load of bacilli in the lung was ascribed to elevated mortality. Interestingly, these discrepant findings have been reported with respect to the alleged effects of PD inhibitors on TB infection. The PD-1/PD-Ls pathway plays an inhibitory role in the lytic degranulation of antigen-specific CD8+ T-cells against MTB. Moreover, MTB antigen induces the upregulation of PD-1 and its ligand(s) in T-cells, B cells, and monocytes in patients with pulmonary TB. ${ }^{13}$ Therefore, suppressing the PD-1/PD-L1 interaction can restore the protective cytokine response by rescuing Th1-like cells from apoptosis induced by the PD-1 pathway. ${ }^{14}$ Recently, Singh et al ${ }^{15}$ found that the locally infiltrated T-cells at the pathological site of miliary tuberculosis are predominantly PD-1+/PD-L1+; this pathway exploited by MTB favors disease dissemination. Moreover, blocking the PD-1/PD-1Ls pathway might enhance the T-cell function toward the microorganisms. Furthermore, in some viral infections, such as chronic hepatitis $B$ virus infection and sepsis model, the administration of PD-1 inhibitors exerted suppressive effects on the pathogens. ${ }^{16,17}$

The current case displayed dominant caseous necrosis after inhibition of the PD-1 pathway, suggesting pronounced inflammation at the specific site, indicating an immune response to microorganisms. However, an excessive immune response leads to overdestruction of the tissue, which is detrimental to the body. Thus, we speculated that increased CD4 and CD8 counts, as well as pulmonary lesions after inhibition of the PD-1 pathway in the current case, indicate active and excessive inflammation against previously latent TB. This phenomenon was similar to the immune reconstitution inflammatory syndrome observed in patients with AIDS after antiretroviral therapy. ${ }^{18}$ Since only a few immune reconstitution inflammatory syndrome cases require steroid use for control, ${ }^{19}$ we speculated that anti-TB agents and a raised number of T-cells might eliminate the microorganisms in a synergistic mechanism. Therefore, we restarted pembrolizumab combined with anti-TB treatment without evidence of TB reactivation. To the best of our knowledge, this is the first report describing the successful use of antitubercular therapy in conjunction with the PD-1 inhibitor. Importantly, the pseudo-progression of TB infection was similar to that observed in some tumors in response to immunotherapeutic agents. ${ }^{20}$ Thus, the abrogation of PD-1/PD-L1 signaling might be beneficial to the TB infection, rather than disadvantages as proposed in previous case reports. ${ }^{3,4}$

\section{Conclusion}

Overall, we presented a melanoma case with TB activated during treatment with the anti-PD-1 monoclonal antibody. The enhanced immune response of T-cells mediated by the application of PD-1 inhibitors might lead to an excessive immune response to TB. Intriguingly, the patient can be safely and effectively treated by combining anti-TB drugs and PD-1 inhibitors. Since additional studies about the reactivation of latent $\mathrm{TB}$ have not yet been reported, TB screening before the initiation of PD-1 inhibitors in all subjects is highly recommended. Thus, further studies on the checkpoint inhibitors in TB-infected patients are essential.

\section{Acknowledgments}

We thank Weipeng Cao, Junfeng Zheng, and Wenwen Li for patient care. We thank Taosheng Ye and other colleagues for help with bronchoscopy. We greatly appreciate the patient and her family for their kind cooperation.

\section{Disclosure}

The authors report no conflicts of interest in this work.

\section{References}

1. Robert C, Schachter J, Long GV, et al. Pembrolizumab versus Ipilimumab in Advanced Melanoma. N Engl J Med. 2015;372(26): 2521-2532.

2. Khoja L, Butler MO, Kang SP, Ebbinghaus S, Joshua AM. Pembrolizumab. J Immunother Cancer. 2015;3:36.

3. Fujita K, TerashimaT, Mio T. Anti-PD1 Antibody Treatment and the Development of Acute Pulmonary Tuberculosis. J Thorac Oncol. 2016;11(12): 2238-2240.

4. Lee JJ, Chan A, Tang T. Tuberculosis reactivation in a patient receiving anti-programmed death-1 (PD-1) inhibitor for relapsed Hodgkin's lymphoma. Acta Oncol. 2016;55(4):519-520.

5. Chu YC, Fang KC, Chen HC, et al. Pericardial Tamponade Caused by a Hypersensitivity Response to Tuberculosis Reactivation after Anti-PD-1 Treatment in a Patient with Advanced Pulmonary Adenocarcinoma. J Thorac Oncol. 2017;12(8):e111-e114.

6. Picchi H, Mateus C, Chouaid C, et al. Infectious complications associated with the use of immune checkpoint inhibitors in oncology: reactivation of tuberculosis after anti PD-1 treatment. Clin Microbiol Infect. 2018;24(3):216-218.

7. Dillman RO, Barth NM, Vandermolen LA, Mahdavi K, Mcclure SE. Should high-dose interleukin-2 still be the preferred treatment for patients with metastatic melanoma? Cancer Biother Radiopharm. 2012; 27(6):337-343.

8. Sharpe AH, Wherry EJ, Ahmed R, Freeman GJ. The function of programmed cell death 1 and its ligands in regulating autoimmunity and infection. Nat Immunol. 2007;8(3):239-245.

9. Lai CC, Lee MT, Lee SH, et al. Risk of incident active tuberculosis and use of corticosteroids. Int J Tuberc Lung Dis. 2015;19(8):936-942.

10. Barber DL, Mayer-Barber KD, Feng CG, Sharpe AH, Sher A. CD4 T cells promote rather than control tuberculosis in the absence of PD-1mediated inhibition. J Immunol. 2011;186(3):1598-1607.

11. Lázár-Molnár E, Chen B, Sweeney KA, et al. Programmed death-1 (PD-1)-deficient mice are extraordinarily sensitive to tuberculosis. Proc Natl Acad Sci U S A. 2010;107(30):13402-13407. 
12. Tousif S, Singh Y, Prasad DV, Sharma P, van Kaer L, das G. T cells from Programmed Death-1 deficient mice respond poorly to Mycobacterium tuberculosis infection. PLoS One. 2011;6(5):e19864.

13. Jurado JO, Alvarez IB, Pasquinelli V, et al. Programmed death (PD)1:PD-ligand 1/PD-ligand 2 pathway inhibits $\mathrm{T}$ cell effector functions during human tuberculosis. J Immunol. 2008;181(1):116-125.

14. Singh A, Mohan A, Dey AB, Mitra DK. Inhibiting the programmed death 1 pathway rescues Mycobacterium tuberculosis-specific interferon $\gamma$-producing $\mathrm{T}$ cells from apoptosis in patients with pulmonary tuberculosis. J Infect Dis. 2013;208(4):603-615.

15. Singh A, Mohan A, Dey AB, Mitra DK. Programmed death $-1^{+}$T cells inhibit effector T cells at the pathological site of miliary tuberculosis. Clin Exp Immunol. 2017;187(2):269-283.

16. Maier H, Isogawa M, Freeman GJ, Chisari FV. PD-1:PD-L1 interactions contribute to the functional suppression of virus-specific CD8+ T lymphocytes in the liver. J Immunol. 2007;178(5):2714-2720.
17. Hotchkiss RS, Monneret G, Payen D. Immunosuppression in sepsis: a novel understanding of the disorder and a new therapeutic approach. Lancet Infect Dis. 2013;13(3):260-268.

18. Lawn SD, Bekker LG, Miller RF. Immune reconstitution disease associated with mycobacterial infections in HIV-infected individuals receiving antiretrovirals. Lancet Infect Dis. 2005;5(6):361-373.

19. Crum-Cianflone NF. Immune reconstitution inflammatory syndromes: what's new? AIDS Read. 2006;16(4):199-206.

20. Wolchok JD, Hoos A, O'Day S, et al. Guidelines for the evaluation of immune therapy activity in solid tumors: immune-related response criteria. Clin Cancer Res. 2009;15(23):7412-7420.

\section{Publish your work in this journal}

OncoTargets and Therapy is an international, peer-reviewed, open access journal focusing on the pathological basis of all cancers, potential targets for therapy and treatment protocols employed to improve the management of cancer patients. The journal also focuses on the impact of management programs and new therapeutic agents and protocols on

\section{Dovepress}

patient perspectives such as quality of life, adherence and satisfaction. The manuscript management system is completely online and includes a very quick and fair peer-review system, which is all easy to use. Visit http://www.dovepress.com/testimonials.php to read real quotes from published authors.

Submit your manuscript here: http://www.dovepress.com/oncotargets-and-therapy-journal 